\title{
Brunnian links
}

\author{
by \\ Paul Gartside (Pittsburgh, PA) and Sina Greenwood (Auckland)
}

\begin{abstract}
A Brunnian link is a set of $n$ linked loops such that every proper sublink is trivial. Simple Brunnian links have a natural algebraic representation. This is used to determine the form, length and number of minimal simple Brunnian links. Braids are used to investigate when two algebraic words represent equivalent simple Brunnian links that differ only in the arrangement of the component loops.
\end{abstract}

1. Introduction. A link with $n$ components, an $n$-link, is the union of $n$ mutually disjoint smooth embeddings of the circle $S^{1}$ in Euclidean 3 -space, $\mathbb{R}^{3}$. An oriented $n$-link is an $n$-link such that each component has a given orientation. Two links, $L_{1}$ and $L_{2}$, are equivalent if there is an ambient isotopy mapping $L_{1}$ onto $L_{2}$. Let $C_{i}$ be the circle $\left\{\langle x, y, 0\rangle: x^{2}+y^{2}=\right.$ $\left.1 /(i+1)^{2}\right\}$ with anti-clockwise orientation. An oriented $n$-link is trivial if it is equivalent to $\mathcal{C}_{n}=\bigcup_{i<n} C_{i}$.

A Brunnian link is a non-trivial $n$-link such that every proper sublink is trivial. The most familiar example is the Borromean rings, a Brunnian 3-link. We will extend the definition of a Brunnian $n$-link to include the trivial $n$-link.

If $L$ is any non-trivial oriented Brunnian $n$-link, then selecting one curve there is an ambient isotopy carrying the remaining curves to $\mathcal{C}_{n-1}$. So $L$ is equivalent to $\mathcal{C}_{n-1} \cup l_{d}$, where $l_{d}$ is a simple closed curve looped around all the curves in $\mathcal{C}_{n-1}$. We will refer to $l_{d}$ as the distinguished curve.

Call a Brunnian $n$-link, $L$, simple if $L$ is equivalent to a link $L^{\prime}=$ $\mathcal{C}_{n-1} \cup\left\{l_{d}\right\}$ such that the projection of $l_{d}$ onto the plane $z=0$ has no self-intersections, meets each ray in the $x y$-plane emanating from the origin exactly once, and has anti-clockwise orientation. See Figure 1(a) for an example.

2000 Mathematics Subject Classification: Primary 57M25; Secondary 20E05.

Key words and phrases: link, Brunnian link, Borromean rings, combinatorial group theory, braid.

Funded by New Zealand Science and Technology Foundation. 

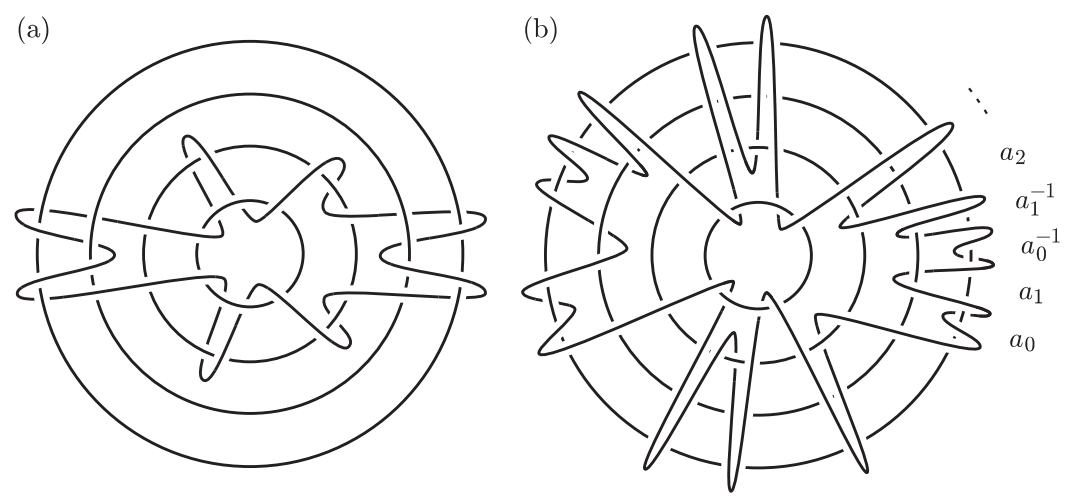

Fig. 1. The simple Brunnian link $a_{0} a_{1} a_{0}^{-1} a_{1}^{-1} a_{2} a_{3} a_{2}^{-1} a_{3}^{-1} a_{1} a_{0} a_{1}^{-1} a_{0}^{-1} a_{3} a_{2} a_{3}^{-1} a_{2}^{-1}$

Any simple Brunnian link is equivalent to a link $L$ of the following type. Let $O=\left\{\langle x, y, z\rangle: x^{2}+y^{2}>1\right\}$. Then $L=\mathcal{C}_{n-1} \cup\left\{l_{d}\right\}$ and the projection of $l_{d}$ onto the plane $z=0$ can be divided into segments with end points in $O$. Following each segment in an anti-clockwise direction it passes over the top of $C_{0}, C_{1}, \ldots, C_{i-1}$, for some $i<n$. It then either passes over the top of $C_{i}$ and back underneath it, or under $C_{i}$ and back over the top of it, before returning over the top of $C_{i-1}, \ldots, C_{0}$ to $O$. Call a Brunnian link of this type canonical. See Figure 1(b) for an example.

The geometric representation of a canonical Brunnian link leads to a natural algebraic representation. We can represent such a link by a word, $a_{i_{0}}^{\varepsilon_{0}} a_{i_{1}}^{\varepsilon_{1}} \ldots a_{i_{m}}^{\varepsilon_{m}}, \varepsilon_{i} \in \mathbb{Z}$. Pick a starting point in $l_{d} \cap O$. If we follow $l_{d}$ in an anti-clockwise direction, $a_{i}$ represents a segment in which $l_{d}$ passes over then under $C_{i}$, and $a_{i}^{-1}$ represents a segment in which $l_{d}$ passes under and back over $C_{i}$. Figures 1(a) and 1(b) are equivalent links represented by

$$
a_{0} a_{1} a_{0}^{-1} a_{1}^{-1} a_{2} a_{3} a_{2}^{-1} a_{3}^{-1} a_{1} a_{0} a_{1}^{-1} a_{0}^{-1} a_{3} a_{2} a_{3}^{-1} a_{2}^{-1} .
$$

Since any simple Brunnian link is equivalent to a canonical Brunnian link, any simple Brunnian link can be represented algebraically by a word. It is immediately clear that such a word is not unique. For example, by choosing a different starting point we will encounter the segments in a different order.

An alternative view of the words introduced above is to recall that the fundamental group of $\mathbb{R}^{3} \backslash \mathcal{C}_{n-1}$ is free with letters represented by simple loops about each circle. These letters correspond to the $a_{i}$ above.

A minimal Brunnian link is a simple Brunnian $n$-link whose associated word is of minimal length (amongst all simple Brunnian $n$-links). Our key results determine the form and length of minimal Brunnian links. In particular, if $n=2^{m}+k$, where $k<2^{m}$, then a minimal Brunnian $n$-link has 
length $2^{m}\left(3 k+2^{m}\right)$. We also compute the number of distinct words associated with minimal Brunnian $n$-links. Finally, we investigate when two words represent topologically equivalent Brunnian links.

The plan for the remainder of the paper is as follows. In the next section the basic definitions and notations are introduced. In Section 3 we determine the length of a minimal Brunnian word, and then minimal Brunnian words are classified and counted. In Section 4 we take a brief look at a generalisation of Brunnian links and determine the form and length of minimal words representing these more general links. In Sections 5 and 6 we use braids to determine when two words represent equivalent Brunnian links.

2. Preliminaries. Denote the set of simple Brunnian $n$-links by $B_{\mathrm{S}}(n)$.

For each $n$ define $A_{n}$ to be the set $\left\{a_{0}, a_{1}, \ldots, a_{n-1}\right\}$, and let $A=\bigcup A_{n}$. Members of $A$ and their inverses will be referred to as letters. A word is any finite sequence $a_{i_{0}}^{\varepsilon_{0}} a_{i_{1}}^{\varepsilon_{1}} \ldots a_{i_{m}}^{\varepsilon_{m}}$ such that $\varepsilon_{i} \in \mathbb{Z}$ for all $i$. Let $e$ denote the empty sequence. We will use the term string when we want to refer to a subsequence in a word even though any such sequence is itself a word. When we refer to an arbitrary string we include the possibility that it is an empty string. We denote words by $v, w$ etc, and strings by $\alpha, \beta$ etc.

If $w$ is a word, denote the set $\left\{a_{i} \in A: a_{i}\right.$ occurs in $\left.w\right\}$ by $A(w)$. A word $w$ is an $n$-word if $A(w)=A_{n}$ or $w=e$. A word $w$ has the form of an n-word if exactly $n$ different letters occur in $w$, or $w=e$ (hence if $w$ has the form of an $n$-word then $A(w)$ need not be $\left.A_{n}\right)$.

Denote the $p$ th occurrence of $a_{i}$ in $w$ by $\stackrel{p}{a}$. If $w$ is a word and $\alpha$ is a string, then $w\left(\alpha / a_{i}\right)$ is the word obtained by replacing every occurrence of $a_{i}^{\varepsilon}$ in $w$ by $\alpha^{\varepsilon}$. We will abbreviate $w\left(e / a_{i}\right)$ to $w\left(-a_{i}\right)$. If $R$ is a set of occurrences of $a_{i}^{ \pm 1}$ in a word $w$, then $w(-R)$ is the string obtained by removing from $w$ the occurrences of $a_{i}^{ \pm 1}$ in the set $R$.

Two words $v, w$ are equivalent if $v$ is convertible into $w$ by a finite sequence of insertions and deletions of subwords $a_{i}^{\varepsilon} a_{i}^{-\varepsilon}$. An $n$-word is reduced if $a_{i}^{\varepsilon} a_{i}^{-\varepsilon}$ does not occur in $w$ for any $i<n$ or $\varepsilon \in \mathbb{Z}$, otherwise $w$ is reducible. If $w$ is a word, let $\varrho(w)$ be the reduced word equivalent to $w$.

We may obtain $\varrho(w)$ from $w$ by successively removing occurrences of $a_{i}^{\varepsilon} a_{i}^{-\varepsilon}$. When $a_{i}^{p} a_{i}^{-\varepsilon}$ is removed we say that $a_{i}^{p}$ cancels with $a_{i}^{-\varepsilon}$. It will be important to know how cancelling proceeds when obtaining a reduced word for expressions like $w\left(-a_{j}\right)$. In some cases an occurrence of $a_{i}$ must cancel with a particular occurrence of $a_{i}^{-1}$, but (by associativity) if cancelling can proceed in different orders there may be several occurrences of $a_{i}^{-1}$ with which $a_{i}$ may cancel. 
An $n$-word $w$ is Brunnian if $w\left(-a_{i}\right)=e$ for each $i<n$. Note that a Brunnian $n$-word represents a Brunnian $(n+1)$-link. Denote the set of Brunnian $n$-words by $B_{\mathrm{A}}(n)$.

Lemma 1. For each $n, B_{\mathrm{A}}(n)$ is a normal subgroup of the free group on $A_{n}$.

Proof. Suppose $v, w \in B_{\mathrm{A}}(n)$. Clearly $w^{-1} \in B_{\mathrm{A}}(n), v w \in B_{\mathrm{A}}(n)$ and $v w v^{-1} \in B_{\mathrm{A}}(n)$. If $w$ is a Brunnian $n$-word and $v$ is equivalent to $w$, then clearly $v$ is a Brunnian $n$-word, and hence $B_{\mathrm{A}}(n)$ is normal.

The length of a word $w$, denoted $l(w)$, is zero if $\varrho(w)=e$, otherwise it is the number of letters in $\varrho(w)$ with exponent 1 or -1 . A Brunnian $n$-word, $w$, is minimal if $l(w) \leq l(v)$ for all $v \in B_{\mathrm{A}}(n)$.

A word $w$ contains a copy of $a_{i_{0}} a_{i_{1}} \ldots a_{i_{m-1}}$ if there are strings $\beta_{0}, \beta_{1}, \ldots, \beta_{m}$ such that $w=\beta_{0} a_{i_{0}} \beta_{1} a_{i_{1}} \ldots a_{i_{m-1}} \beta_{m}$. A Brunnian $n$-word, $w$, is basic if it does not contain a copy of a word having the form of a Brunnian $n$-word other than $w$ itself.

The following properties are immediately obvious from the definition of a Brunnian $n$-word.

1. Let $w$ be a Brunnian $n$-word, $n>1$. Then:

(a) if $w=\alpha a_{i} \beta$ and $w$ is reduced, then $\beta$ contains a copy of $\alpha^{-1}$;

(b) $a_{i}^{-1}$ occurs in $w$ exactly the same number of times as $a_{i}$;

(c) if $w \neq e$ then for each $i<n$ there exist $\varepsilon, \varepsilon^{\prime} \in \mathbb{Z}$ and $j<n$ such that $a_{j}^{\varepsilon} a_{i}^{\varepsilon^{\prime}} a_{j}^{-\varepsilon}$ occurs in $w$

(d) if $w \neq e$ then for each $i<n$ there is a non-empty string $\varphi$ such that $a_{i} \notin A(\varphi)$ and $a_{i} \varphi a_{i}^{-1}$ occurs in $w$;

(e) if $w$ is basic then $w \neq a_{i} \varphi a_{i}^{-1}$ for any string $\varphi$;

(f) $\varrho\left(\left(w\left(-a_{i}\right)\right)\left(-a_{j}\right)\right)=\varrho\left(\left(w\left(-a_{j}\right)\right)\left(-a_{i}\right)\right)=e$ for every $i, j<n$ for every $i, j<n$ (by associativity).

2. $w$ is a Brunnian $n$-word if and only if $a_{i} w a_{i}^{-1}$ is a Brunnian $n$-word for all $i<n$.

Property 2 is equivalent to: $\alpha \beta$ is a Brunnian $n$-word if and only if $\beta \alpha$ is a Brunnian $n$-word. Of course $\alpha \beta$ and $\beta \alpha$ represent the same Brunnian link. They simply relate to different starting points.

This relation is important when deducing how words are formed from subwords. Let $\sim_{\mathrm{S}}$ be the conjugacy equivalence relation over $B_{\mathrm{A}}(n): w \sim_{\mathrm{S}} v$ if and only if there exist strings $\alpha$ and $\beta$ such that $w=\alpha \beta$ and $v=\beta \alpha$.

We introduce another equivalence relation which will help to simplify proofs in Section 3. Many of these proofs involve certain types of strings which occur in a given word. These strings may take a variety of forms de- 
pending on whether the occurrence of each letter has index \pm 1 . To prove these lemmas for each one of the different forms involves repetitious arguments. In order to circumvent such repetition we define $\alpha \equiv \beta$ if and only if there is a sequence $\alpha=\alpha_{0}, \alpha_{1}, \ldots, \alpha_{m}=\beta$ such that for each $i$ we have $\alpha_{i+1}=\alpha_{i}\left(a_{j}^{-1} / a_{j}\right)$ for some $j$. We will prove each lemma for one case (one element of the relevant $\equiv$ equivalence class) whenever it is clear that the other cases follow a similar argument.

The following is a natural extension of the notion of a Brunnian word. An $m n$-word, $m \geq n$, is a word $w$ such that $w$ is an $m$-word and $w\left(-a_{i}\right)=e$ for all $i<n$. Extending our results on Brunnian words, we will compute the form and length of minimal $m n$-words.

3. Minimal Brunnian words. In this section we determine the length, form and number of minimal Brunnian $n$-words. We require several technical lemmas.

Lemma 2. Suppose $w$ is a reduced Brunnian $n$-word, $n>1$, and suppose $j<n$. Pick integers $i, r, s$ and $\varepsilon$, and strings $\alpha$ and $\beta$ such that, modulo $\equiv$,

$$
w=\alpha a_{i}^{r} a_{j}^{\varepsilon}\left(a_{i}^{-1}\right) \beta .
$$

Partition all the occurrences of $a_{i}^{ \pm 1}$ into the maximum number of components such that if $k \neq i$ or $j$, then any member of any component can only cancel with another member of the same component in the reduction of $w\left(-a_{k}\right)$. Let $R$ be the component containing $\stackrel{r}{a}$, and $S$ the union of all the other components. Let $v_{1}=\varrho(w(-R))$ and $v_{2}=\varrho(w(-S))$. Let $u_{R}^{\prime}$ and $u_{S}^{\prime}$ be the words obtained from $w\left(-a_{j}\right)$ by replacing with $\left(a_{j} a_{i}^{-1} a_{j}^{-1}\right)^{ \pm 1}$ each occurrence of $a_{i}^{ \pm 1}$ in $R$, for $u_{R}^{\prime}$, and each occurrence of $a_{i}^{ \pm 1}$ in $S$, for $u_{S}^{\prime}$. Let $u_{R}=\varrho\left(u_{R}^{\prime}\right)$ and $u_{S}=\varrho\left(u_{S}^{\prime}\right)$. Then

(i) $a_{i}^{s} \notin R$.

If $w$ is minimal then

(ii) $v_{l}\left(-a_{j}\right) \neq e$ whenever $v_{l} \neq e, l=1,2$.

If $a_{j}$ occurs at least as frequently as $a_{i}$ in $w$, then:

(iii) either $v_{1} \neq e$ or $v_{2} \neq e$;

(iv) $|R|=|S|$, and $S$ is a single component;

(v) $a_{i}$ occurs the same number of times in $w$ as $a_{j}$;

(vi) $l\left(u_{R}\right)=l\left(u_{S}\right)=l(w)$.

Proof. (i) If $a_{i}^{-1} \in R$ then there is a sequence

$$
\stackrel{r}{a_{i}}=\stackrel{p_{0}}{a_{i}},{\stackrel{p}{p_{1}}}_{i}^{-1}, \ldots, \vec{p}_{i}^{-1}=a_{i}^{-1},
$$


such that for all $h$ there is a $k \neq i, j$ such that $a_{i}^{a_{h}}$ cancels with $a_{i}^{-\varepsilon}$ in the reduction of $w\left(-a_{k}\right)$.

Hence $a_{j}$ occurs the same number of times as $a_{j}^{-1}$ between each pair $\begin{gathered}p_{h} \\ a_{i}^{\varepsilon}\end{gathered}$ $p_{h+1}$

and $a_{i}^{-\varepsilon}$. It follows easily, by induction, that $a_{j}$ and $a_{j}^{-1}$ occur the same number of times between $\stackrel{r}{a}$ ind any other member of the sequence. This is not the case, however, for $\stackrel{r}{a_{i}}$ and $a_{i}^{-1}$. Hence $a_{i}^{-1} \notin R$.

(ii) Suppose $v_{1} \neq e$. If $\varrho\left(v_{1}\left(-a_{j}\right)\right)=e$ then $v_{1}$ is an $n$-word since by associativity, for each $k \neq i, j$ members of $S$ cancel with each other in the reduction of $v_{1}\left(-a_{k}\right)$ so that $\varrho\left(v_{1}\left(-a_{k}\right)\right)=e$. And clearly $\varrho\left(v_{1}\left(-a_{i}\right)\right)=e$. Thus $v_{1}$ is a copy of an $n$-word in $w$, but $v_{1} \neq w$, hence $\varrho\left(v_{1}\left(-a_{j}\right)\right) \neq e$.

(iii) Suppose $v_{1}=v_{2}=e$. Let $w_{1}$ be the word obtained from $w$ by replacing each occurrence of $a_{i}^{ \pm 1}$ in $R$ by $a_{n}^{ \pm 1}$.

If $\varrho\left(w_{1}\left(-a_{j}\right)\right) \neq e$ then $\varrho\left(w_{1}\left(-a_{j}\right)\right)$ has the form of a Brunnian $n$-word since for each $k \neq i, j, \varrho\left(w_{1}\left(-a_{k}\right)\right)=\varrho\left(w\left(-a_{k}\right)\right)=e, \varrho\left(w_{1}\left(-a_{n}\right)\right)=v_{1}=e$, and $\varrho\left(w_{1}\left(-a_{i}\right)\right)=e$ because $v_{2}=e$. Since $l\left(\varrho\left(w_{1}\left(-a_{j}\right)\right)\right)<l(w)$ we have a contradiction, and hence $\varrho\left(w_{1}\left(-a_{j}\right)\right)=e$. Thus $w_{1}$ is an $(n+1)$-word.

Pick $a_{j 1}$ and $a_{i 1}$, and components $R_{1}$ and $S_{1}$ of $w_{1}$ analogously to $R$ and $S$ in $w$ (pick one of the most frequently occurring letters for $a_{j 1}$ ). If $v_{11}=$ $\varrho\left(w_{1}\left(-R_{1}\right)\right)=e$ and $v_{12}=\varrho\left(w_{1}\left(-S_{1}\right)\right)=e$, obtain $u$ by replacing each $a_{i 1}^{ \pm 1}$ in $R_{1}$ by $a_{n+1}^{ \pm 1}$ and reducing. If $\varrho\left(u\left(-a_{j 1}\right)\right) \neq e$ then let $w_{2}=\varrho\left(v\left(-a_{j 1}\right)\right)$, otherwise let $w_{2}=u$.

If $v_{11} \neq e$ and $\varrho\left(v_{11}\left(-a_{j}\right)\right)=e$, let $w_{2}=v_{11}$. It is easy to show that $w_{2}$ is an $(n+1)$-word. If $v_{11} \neq e$ and $\varrho\left(v_{11}\left(-a_{j}\right)\right) \neq e$, let $w_{2}=\varrho\left(v_{11}\left(-a_{j}\right)\right)$. Continue by induction. For some $m$ we must obtain a word $w_{m}$, corresponding to a word in $w$ that has the form of a Brunnian $n$-word. Since $l\left(w_{m}\right)<l(w)$ we have a contradiction and hence either $v_{1}=e$ or $v_{2}=e$.

(iv), (v) and (vi). We first prove

Claim. $u_{R} \neq e \neq u_{S}$ and $u_{R}$ and $u_{S}$ are Brunnian $n$-words.

Proof. We first show that $u_{R} \neq e \neq u_{S}$. If $a_{i}^{t}$ in $u_{R}^{\prime}$ is part of a string $t^{\prime}$

that replaced a member of $R$ in $w$, and $a_{i}^{-\varepsilon}$ in $u_{R}^{\prime}$ was a member of $S$ in $w$, then $a_{j}^{ \pm 1}$ must occur an odd number of times between $a_{i}^{\varepsilon}$ and $a_{i}^{t^{\prime}}$, twice for each member from $R$ that occurs between them, and once beside $a_{i}^{\epsilon}$. Hence when cancelling to obtain $u_{R}$, members of $R$ must cancel with each other and members of $S$ must cancel with each other. Members of $R$ cancel to obtain $\varrho\left(w\left(-a_{j}\right)\right)$ if and only if the corresponding strings $a_{j}^{ \pm \varepsilon} a_{i}^{ \pm \varepsilon} a_{j}^{\mp \varepsilon}$ cancel when $u_{R}^{\prime}$ is reduced. Hence if $u_{R}=e$, then $\varrho\left(w\left(-a_{j}\right)(-R)\right)=e$ and 
$\varrho\left(w\left(-a_{j}\right)(-S)\right)=e$. But since either $\varrho\left(v_{1}\left(-a_{j}\right)\right) \neq e$ or $\varrho\left(v_{2}\left(-a_{j}\right)\right) \neq e$ by (ii) and (iii), $u_{R} \neq e$. Similarly, $u_{S} \neq e$.

We now show that $u_{R}$ and $u_{S}$ are Brunnian $n$-words. We have

$$
\varrho\left(u_{R}\left(-a_{j}\right)\right)=\varrho\left(w\left(-a_{j}\right)\right)=e, \quad \varrho\left(u_{R}\left(-a_{i}\right)\right)=\varrho\left(w\left(-a_{j}\right)\left(-a_{i}\right)\right)=e .
$$

Suppose $k \neq i, j$ and consider $u_{R}\left(-a_{k}\right)$. Since members of $R$ need only cancel with members of $R$ in the reduction of $w\left(-a_{k}\right)$ and hence in the reduction of $w\left(-a_{j}\right)\left(-a_{k}\right)$, it follows that the strings $a_{j}^{ \pm \varepsilon} a_{i}^{ \pm \varepsilon} a_{j}^{\mp \varepsilon}$ cancel with each other and

$$
\varrho\left(u_{R}\left(-a_{k}\right)\right)=\varrho\left(w\left(-a_{k}\right)\left(-a_{j}\right)\right)=e .
$$

We may argue similarly for $u_{S}$. Thus $u_{R}$ and $u_{S}$ are Brunnian $n$-words, and the claim is proved.

The number of $a_{j}^{ \pm 1}$ 's occurring in $u_{R}$ is at most $2|R|$, and in $u_{S}$ at most $2|S|$. If $a_{j}^{ \pm 1}$ occurs $J$ times in $w$ then $J \geq|R|+|S|$, since $a_{j}$ occurs at least as often as $a_{i}$ in $w$. Since $w$ is minimal, $l\left(u_{R}\right) \geq l(w)$ and $l\left(u_{S}\right) \geq l(w)$, but if $l\left(u_{R}\right)>l(w)$ then $l\left(u_{S}\right)<l(w)$, and if $l\left(u_{S}\right)>l(w)$, then $l\left(u_{R}\right)<l(w)$. Hence $a_{j}$ occurs $2|S|=2|R|$ times, $|S|=|R|$, and $S$ is a single component.

Lemma 3. Suppose $w$ is a minimal Brunnian $n$-word in which a particular collection of letters only appear in strings $\alpha^{ \pm 1}$ where $\alpha$ is a Brunnian word, $\alpha a_{j}^{\varepsilon} \alpha^{-1}$ occurs in $w$, and $a_{j}$ does not occur less frequently than $\alpha$. Then it is possible to replace exactly half the occurrences of $\alpha^{ \pm 1}$ in $w\left(-a_{j}\right)$ by $a_{j}^{ \pm 1} \alpha^{ \pm 1} a_{j}^{\mp 1}$, and reduce to obtain an $n$-word that is also minimal.

Proof. The proof of Lemma 2 can easily be adjusted to this situation.

LEMMA 4. If $w$ is a minimal Brunnian $n$-word then for each $i<n$ and $\varepsilon= \pm 1, a_{i}^{\varepsilon}$ does not occur consecutively in $w$.

Proof. Obvious if $n \leq 2$. Suppose $n>2$ and $w=a_{i} a_{i} \alpha$ (it is sufficient to obtain a contradiction for this case). Partition the occurrences of $a_{i}^{ \pm 1}$ in $w$ such that members of each component can only cancel with each other in $\varrho\left(w\left(-a_{j}\right)\right)$ for each $j \neq i$. If $\stackrel{1}{a}$ and $\stackrel{2}{a}$ are in the same component, then

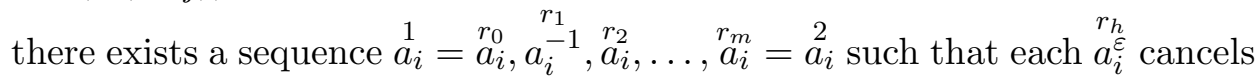
$r_{h+1}$ with $a_{i}^{-\varepsilon}$ in $\varrho\left(w\left(-a_{j}\right)\right)$ for some $j$. Then $a_{i}$ and $a_{i}^{-1}$ occur an equal number of times between each pair $a_{i}^{\varepsilon}$ and $a_{i}^{-\varepsilon}$.

Let $n_{h}$ be the total number of occurrences of $a_{i}^{ \pm 1}$ between $a_{i}$ and $a_{i}^{\varepsilon}$. A simple induction argument shows that $n_{h}$ is always even if $\varepsilon=-1$ and odd if $\varepsilon=1$. But $a_{i}^{-1}$ cancels with $\stackrel{2}{a}_{i}$ so there must be an even number 
between ${ }_{i}^{r_{m-1}^{-1}}$ and $\stackrel{2}{a_{i}}$. However, there are $n_{m}-1$ occurrences between $a_{i}^{r_{m-1}}$ and $\stackrel{2}{a}$, giving a contradiction.

Lemma 5. For each $n>1$, if $w$ is a minimal Brunnian n-word, then there is a Brunnian n-word $v$ such that:

(i) all letters occur the same number of times in $v$ as in $w$;

(ii) if $n$ is even, then all letters in $v$ occur in strings $a_{i} a_{j} a_{i}^{-1} a_{j}^{-1}$ (modulo $\equiv)$, and for any two such strings $\alpha$ and $\beta$, either $A(\alpha)=A(\beta)$ or $A(\alpha) \cap A(\beta)=\emptyset$; if $n$ is odd, then all letters except one of the least occurring letters, $a_{k}$ say, occur in such strings, and $a_{k}$ only occurs in strings $a_{k}\left(a_{i} a_{j} a_{i}^{-1} a_{j}^{-1}\right) a_{k}^{-1}\left(a_{i} a_{j} a_{i}^{-1} a_{j}^{-1}\right)^{-1}$ (modulo $\equiv$, modulo $\left.\sim_{\mathrm{S}}\right)$.

Proof. By Lemma 2(v),(vi) and Lemma 4, obtain from $w$ a minimal Brunnian $n$-word $u$ such that one of the most frequently occurring letters, $a_{j}^{ \pm 1}$, only occurs in strings $a_{j}^{\varepsilon} a_{i}^{\varepsilon} a_{j}^{-\varepsilon}, \varepsilon= \pm 1$, and $a_{i}^{ \pm 1}$ and $a_{j}^{ \pm 1}$ occur the same number of times in $u$ as in $w$. Pick one of the strings $a_{j}^{\varepsilon} a_{i}^{\varepsilon} a_{j}^{\frac{y}{\varepsilon}}$ occurring in $u$. Partition the occurrences of $a_{j}$ in $u$ into a maximal number of components such that members of each component can only cancel with members of the same component in the reduction of $u\left(-a_{k}\right)$ if $k \neq i, j$. Let $X$ be the component containing $a_{j}^{\varepsilon}$ and $Y$ the component containing $a_{j}^{-\varepsilon}$. By arguing as in the proof of Lemma 2(i) we can deduce that for each string $a_{j}^{\varepsilon} a_{i}^{\varepsilon} a_{j}^{-\varepsilon}$ occurring in $u$, one of $a_{j}^{ \pm 1}$ is in $X$ and the other is in $Y$.

Let $u_{1}$ be the word obtained from $u$ by removing every occurrence of $a_{i}$ and replacing every member $a_{j}^{ \pm \varepsilon}$ of $X$ by $a_{i}^{\varepsilon} a_{j}^{\varepsilon} a_{i}^{-\varepsilon}$. Then the letters $a_{i}^{ \pm 1}$ and $a_{j}^{ \pm 1}$ only occur in $u_{1}$ as part of a string $a_{i} a_{j} a_{i}^{-1} a_{j}^{-1}$ (modulo $\equiv$ ), and by Lemma $2(\mathrm{vi}), l(u)=l\left(u_{1}\right)$.

Suppose $a_{k}$ is one of the most frequently occurring letters other than $a_{i}$ or $a_{j}$. Pick $a_{l}$ such that $a_{l}^{\varepsilon} a_{k}^{\varepsilon} a_{l}^{-\varepsilon}$ occurs. As above, obtain $u_{2}$ such that $a_{k}^{ \pm 1}$ and $a_{l}^{ \pm 1}$ only occur in strings $\left(a_{k} a_{l} a_{k}^{-1} a_{l}^{-1}\right)^{ \pm 1}$.

Suppose $i=l$. Then all occurrences of $a_{i}^{ \pm 1}, a_{j}^{ \pm 1}$ and $a_{k}^{ \pm 1}$ are (modulo $\equiv$ ) in strings $\alpha^{ \pm 1}$ and $\beta^{ \pm 1}$, where

$$
\alpha=a_{i} a_{j} a_{i}^{-1} a_{j}^{-1} \text { and } \beta=a_{k} a_{i} a_{k}^{-1} a_{i}^{-1} a_{j} a_{i} a_{k} a_{i}^{-1} a_{k}^{-1} a_{j}^{-1} \text {. }
$$

Let $u^{\prime}=u_{2}\left(a_{k} a_{i} a_{j} a_{i}^{-1} a_{j}^{-1} a_{k}^{-1} / \beta\right)$. Clearly, $\varrho(v) \neq e$, and it is not too difficult to show that $v$ is an $n$-word. Once again we have a contradiction, since $u^{\prime}$ is shorter than $v_{2}$, and so $l \neq i$. Similarly $l \neq j$.

Continue by induction to obtain a Brunnian $n$-word $u_{m}$ in which all the letters, except one if $n$ is odd, only occur in strings $a_{i} a_{j} a_{i}^{-1} a_{j}^{-1}$ (modulo $\equiv$ ), and for any two such strings $\alpha$ and $\beta$, either $A(\alpha)=A(\beta)$ or $A(\alpha) \cap A(\beta)=\emptyset$. 
If $n$ is even we are done. If $n$ is odd, let $a_{k}$ be the only letter that does not occur in such a string. Pick $\alpha$ such that $\alpha a_{k} \alpha^{-1}$ occurs in $u_{m}$. Then $a_{k}$ does not occur less often than $\alpha$ in $u_{m}$ (otherwise swap the occurrences of $\alpha^{ \pm 1}$ with those of $a_{k}^{ \pm 1}$ to obtain a Brunnian $n$-word shorter than $\left.w\right)$. By Lemma 3 we are done.

3.1. Length. We now determine the length of a minimal Brunnian $n$ word. We first determine an upper bound.

Lemma 6. Suppose $n=2^{m}+k, k<2^{m}$ and $n>0$. Then there is a Brunnian $n$-word, $w_{n}$, such that $l\left(w_{n}\right)=2^{m}\left(3 k+2^{m}\right), 2^{m}-k$ letters occur $2^{m}$ times and $2 k$ letters occur $2^{m+1}$ times in $w_{n}$.

Proof. Let $w_{0}=a_{0}$, a Brunnian 1-word. Suppose $n=2^{m}+k, k<2^{m}$ and $w_{n}$ is a Brunnian $n$-word such that:

- $w_{n}$ has length $2^{m}\left(3 k+2^{m}\right)$;

- $2^{m}-k$ letters occur $2^{m}$ times and $2 k$ letters occur $2^{m+1}$ times.

Pick one of the letters $a_{i}$ that occurs $2^{m}$ times and let

$$
w_{n+1}=w_{n}\left(a_{i} a_{n} a_{i}^{-1} a_{n}^{-1} / a_{i}\right) .
$$

Then $2^{m}-(k+1)$ letters occur $2^{m}$ times in $w_{n+1}$, and since both $a_{i}^{ \pm 1}$ and $a_{n}^{ \pm 1}$ occur twice as often in $w_{n+1}$ as $a_{i}$ occurred in $w_{n}$, there are $2 k+2$ letters occurring $2^{m+1}$ times in $w_{n+1}$. Also, $l\left(w_{n+1}\right)=l\left(w_{n}\right)+3 \cdot 2^{m}=$ $2^{m}\left(3(k+1)+2^{m}\right)$. If $k+1=2^{m}$, then $l\left(w_{n+1}\right)=2^{m+1} 2^{m+1}$.

Theorem 7. If $w$ is a minimal Brunnian $n$-word, $0<n=2^{m}+k$ and $k<2^{m}$, then $l(w)=2^{m}\left(3 k+2^{m}\right)$.

Proof. Clearly the theorem holds when $n=1$. Suppose that it holds for each $n^{\prime}<n$. If $n$ is even, by Lemma 5 pick a minimal Brunnian $n$-word, $w$, such that the letters only occur in strings $\alpha_{t}^{ \pm 1}$ where $\alpha_{t}=$ $a_{t} a_{n-(t+1)} a_{t}^{-1} a_{n-(t+1)}^{-1}, 0 \leq t<n / 2$. Let $v$ be the word obtained by replacing each $\alpha_{t}$ by $a_{t}$. Then $v$ is an $n / 2$-word. Clearly $w$ is minimal if and only if $v$ is minimal, and $l(w)=4 l(v)=4 \cdot 2^{m-1}\left(3 k / 2+2^{m-1}\right)=$ $2^{m}\left(3 k+2^{m}\right)$.

Suppose $n$ is odd. Pick a minimal Brunnian $n$-word, $w$, such that all the letters other than $a_{0}$ only occur in strings $\alpha_{t}^{ \pm 1}$, where $\alpha_{t}=a_{t} a_{n-t} a_{t}^{-1} a_{n-t}^{-1}$, $0<t<(n-1) / 2$, and $a_{0}$ only occurs in strings $\beta=a_{0} \alpha_{1} a_{0}^{-1} \alpha_{1}^{-1}$. Suppose that the number of occurrences of the $\alpha_{t}^{ \pm 1}$ 's in $w$ is $x$, and $\beta^{ \pm 1}$ occurs $y$ times. Obtain a Brunnian word $u$ from $w$ by replacing each $\alpha_{i}^{ \pm 1}$ by $a_{i}^{ \pm 1}$, and each $\beta^{ \pm 1}$ by $a_{0}^{ \pm 1}$. Then $u$ has the form of a Brunnian $(n-1) / 2$-word. Obtain a word $v$ from $w$ with the form of a Brunnian $(n+1) / 2$-word, by replacing 
each $\alpha_{i}^{ \pm 1}$ by $a_{i}^{ \pm 1}$. Then

$$
\begin{aligned}
& l(w)=4 x+10 y \leq 2^{m}\left(3 k+2^{m}\right), \\
& l(u)=x+y \geq 2^{m-1}\left(3\left(\frac{k-1}{2}\right)+2^{m-1}\right), \\
& l(v)=x+4 y \geq 2^{m-1}\left(3\left(\frac{k+1}{2}\right)+2^{m-1}\right),
\end{aligned}
$$

and hence

$$
2^{m}\left(3 k+2^{m}\right) \geq 4 x+10 y=2(l(u)+l(v)) \geq 2^{m}\left(3 k+2^{m}\right) .
$$

Hence $l(w)=2^{m}\left(3 k+2^{m}\right)$.

3.2. Form. We can now establish that any minimal Brunnian word must have a certain form.

Let $\mathcal{M}^{\prime}$ be the collection of words such that:

(i) $\forall a_{i} \in A, a_{i}, a_{i}^{-1} \in \mathcal{M}^{\prime}$;

(ii) $\forall v, w \in \mathcal{M}^{\prime}$, if $A(v) \cap A(w)=\emptyset$ then $v w v^{-1} w^{-1} \in \mathcal{M}^{\prime}$;

(iii) $\forall w \in \mathcal{M}^{\prime}$, if $v \sim_{\mathrm{S}} w$ then $v \in \mathcal{M}^{\prime}$,

and let

$$
\mathcal{M}=\left\{w \in \mathcal{M}^{\prime}:(\exists n \in \omega) A(w)=A_{n}\right\} .
$$

Observe that every $w \in \mathcal{M}$ is $\sim_{\mathrm{S}}$ equivalent to a string of the form $\alpha \beta \alpha^{-1} \beta^{-1}$ (provided $l(w)>1$ ). Also note that every word in $\mathcal{M}$ is a basic Brunnian word. Finally, we remark that not all Brunnian words have this form (for example, $a_{0} a_{1} a_{0}^{-1} a_{2} a_{0} a_{1}^{-1} a_{0}^{-1} a_{0}^{-1} a_{1} a_{0} a_{2}^{-1} a_{0}^{-1} a_{1}^{-1} a_{0}$ ).

TheOREM 8. Every minimal Brunnian $n$-word $w$ is in $\mathcal{M}$.

Proof. We will assume that all strings in this proof are chosen modulo $\equiv$. We prove this by induction on $n$. If $w$ is a minimal Brunnian 1-word then $w=a_{0}^{ \pm 1} \in \mathcal{M}$. If $w$ is a minimal Brunnian 2-word then $w$ is a non-trivial arrangement of the letters $a_{0}, a_{0}^{-1}, a_{1}$ and $a_{1}^{-1}$ and hence $w \in \mathcal{M}$. If $w$ is a minimal 3-word then one letter occurs twice. Hence $w$ has the form $a_{0} \alpha a_{0}^{-1} \alpha^{-1}$ and clearly $\alpha=a_{1} a_{2} a_{1}^{-1} a_{2}^{-1}$.

Suppose for each $n^{\prime}<n$ any minimal $n^{\prime}$-word is a member of $\mathcal{M}$, and $n>3$.

Suppose $a_{j}$ is one of the most frequently occurring letters and $a_{i} a_{j} a_{i}^{-1}$ occurs in $w$. By Lemma 2(ii),(iii) and Lemma 6, remove half the occurrences of $a_{i}$ from $w\left(-a_{j}\right)$ and reduce to obtain a minimal $(n-1)$-word $v$. Then $v=\alpha \beta \alpha^{-1} \beta^{-1}$. We may assume that $a_{i}^{ \pm 1}$ occurs in $\alpha$ and not in $\beta$. Pick one of the most frequently occurring letters $a_{k}$ in $\beta$, which only occurs in strings $a_{k} a_{l} a_{k}^{-1} a_{l}^{-1}$ (which is possible since $n>3$ ). At least one string $a_{l} a_{k} a_{l}^{-1}$ corresponds to such a string in $w$. If not then $\varrho\left(w\left(-a_{h}\right)\right) \neq e$ for $h=i, l$ or $k$, or $a_{l}$ must occur consecutively in $w$, contradicting Lemma 4 . Remove 
half the occurrences of $a_{l}$ from $w\left(-a_{k}\right)$ and reduce to obtain a minimal $(n-1)$-word $v^{\prime}$. Now $v^{\prime}=\alpha^{\prime} \beta^{\prime}\left(\alpha^{\prime}\right)^{-1}\left(\beta^{\prime}\right)^{-1}, a_{i}^{ \pm 1}$ and $a_{j}^{ \pm 1}$ only occur in $\alpha^{\prime}$ say, and they only occur in strings $a_{i} a_{j} a_{i}^{-1} a_{j}^{-1}$. Hence it is clear that $w=\alpha^{\prime} \beta\left(\alpha^{\prime}\right)^{-1} \beta^{-1} \in \mathcal{M}$.

3.3. Number. In this section we establish the number of different forms of a minimal Brunnian $n$-word. We consider the words $v, w \in \mathcal{M}$ to have the same form if there is a sequence $u_{0}, u_{1}, \ldots, u_{m}$ such that $u_{0}=v, u_{m}=w$ and for each $h<m$ either $u_{h} \equiv u_{h+1}, u_{h+1}$ may be obtained from $u_{h}$ by interchanging all occurrences of $a_{i}^{ \pm 1}$ with $a_{j}^{ \pm 1}$ for some $i, j<n$, or $u_{h+1}=$ $u_{h}(\beta / \alpha)$ where $\alpha \sim_{\mathrm{S}} \gamma \delta \gamma^{-1} \delta^{-1}, \alpha$ occurs in $u_{h}$ and $\beta \sim_{\mathrm{S}} \alpha$. We associate members of $\mathcal{M}$ with binary trees, so that the number of forms of a word is the number of non-isomorphic trees that are associated with minimal $n$-words.

For each $w \in \mathcal{M}$ construct a tree $T_{w}$ such that the root is the word $w$, if a member of $T_{w}$ is a string of length 1 then it has no successors, and if it is a string $\sigma$ and $\sigma \sim_{\mathrm{S}} \alpha \beta \alpha^{-1} \beta^{-1}$, then it has two successors, $\alpha$ and $\beta$. Observe that:

- $T_{w}$ is unique up to isomorphism;

- if $w \in \mathcal{M}$ and $|A(w)|=n$, then $T_{w}$ has $n$ leaves (members of $T$ with no successors);

- $v$ and $w$ have the same form if and only if $T_{v}$ and $T_{w}$ are isomorphic.

Let $f(m, 0)=f(m, 1)=f\left(m, 2^{m}\right)=1$ for every $m$. Define recursively, for $m>1$ and $k<2^{m}$,

$$
f(m, k)= \begin{cases}\sum_{l=0}^{\lfloor k / 2\rfloor} f(m-1, l) \cdot f(m-1, k-l) & \text { if } k \leq 2^{m-1}, \\ \sum_{l=k-2^{m-1}}^{\lfloor k / 2\rfloor} f(m-1, l) \cdot f(m-1, k-l) & \text { if } k>2^{m-1} .\end{cases}
$$

Theorem 9. Let $n=2^{m}+k$ where $k<2^{m}$. The number of forms of a minimal Brunnian $n$-word is $f(m, k)$.

Proof. The number of forms is the number of non-isomorphic binary trees with $2^{m}-k$ leaves at height $m$, and $2 k$ leaves at height $k+1$. Suppose $T$ is a finite binary tree, and $l$ and $r$ are the two immediate successors of the root of $T$. Let $L(T)$ be the subtree of all successors of $l$ including $l$, and $R(T)$ the subtree of all successors of $r$ including $r$. Then two trees $S$ and $T$ are isomorphic if and only if $L(S)$ is isomorphic to $L(T)$ and $R(S)$ is isomorphic to $R(T)$, or $L(S)$ is isomorphic to $R(T)$ and $R(S)$ is isomorphic to $L(T)$. The number of such non-isomorphic trees is $f(m, k)$. 
We may also use the binary trees to determine the length of members of $\mathcal{M}$ that have particular forms. Let $\mathcal{B}$ be the isomorphism classes of $\left\{T_{w}: w \in \mathcal{M}\right\}$. We will simply speak of the element $T$ in $\mathcal{B}$ rather than the equivalence classe $[T]$. Now define $l^{\prime}: \mathcal{B} \rightarrow \omega$ as follows. Given $T \in \mathcal{B}$ assign each leaf the number 1 . Each vertex is assigned the number equal to twice the sum of the numbers assigned to its immediate successors. Then $l^{\prime}(T)$ is the number assigned to the root of $T$. It is not difficult to conclude that $l(w)=l^{\prime}\left(T_{w}\right)$. Note that $l^{\prime}$ is well defined but not 1-1.

One can now easily prove:

LEMMA 10. The maximum length of an $n$-word in $\mathcal{M}$ is $2^{n}+2^{n-2}+$ $2^{n-3}+\cdots+2$.

4. $m n$-words. We now address the minimal length of an $m n$-word, first establishing the form of a minimal $m n$-word.

LEMma 11. If $0<n \leq m$ then any minimal $m n$-word has the form

$$
a_{i_{n}}^{\varepsilon_{n}} a_{i_{n+1}}^{\varepsilon_{n+1}} \ldots a_{i_{m-1}}^{\varepsilon_{m-1}} u a_{a_{m-1}}^{-\varepsilon_{m-1}} \ldots a_{a_{n}}^{-\varepsilon_{n}}
$$

where each $\varepsilon_{h}= \pm 1$, and $u$ is a minimal Brunnian n-word.

Proof. Let $w$ be a minimal $m n$-word which is not an $m n^{\prime}$-word for any $n^{\prime}>n$. When $m=1$ the claim is obvious. So we argue by induction on $m$.

Suppose $w$ is a minimal $m n$-word and any minimal $r n$-word has the required form if $r<m$. Let $w^{\prime}=\varrho\left(w\left(-a_{m-1}\right)\right)$. If $w$ is also an $m n^{\prime}$-word we are done. It might not be an $m^{\prime} n^{\prime}$-word, but if we rename the letters appropriately, we can ensure that it is. Hence, without loss of generality, assume that for some $n \leq n^{\prime} \leq m^{\prime}<m, w^{\prime}$ is an $m^{\prime} n^{\prime}$-word but not an $m^{\prime}\left(n^{\prime}-1\right)$-word. Then $v=a_{m^{\prime}} a_{m^{\prime}+1} \ldots a_{m-1} w^{\prime}\left(-a_{m-1}\right) a_{m-1}^{-1} \ldots a_{m^{\prime}}^{-1}$ is an $m n^{\prime}$-word, and $l(v) \leq l(w)$ since the minimum collection of letters removed from $w$ in $w^{\prime}$ is one occurrence of each of $a_{m^{\prime}}^{ \pm 1}, \ldots, a_{m-1}^{ \pm 1}$. It follows that $w^{\prime}$ is a minimal $m^{\prime} n^{\prime}$-word

$$
a_{i_{n}^{\prime}}^{\varepsilon_{n}} a_{i_{n+1}}^{\varepsilon_{n+1}} \ldots a_{i_{m^{\prime}-1}}^{\varepsilon_{m-1}} u a_{a_{m^{\prime}-1}}^{-\varepsilon_{m-1}} \ldots a_{a_{n}^{\prime}}^{-\varepsilon_{n}}
$$

where $u$ is a minimal $n^{\prime}$-word, and exactly one occurrence of each of $a_{m^{\prime}}^{ \pm 1}, \ldots, a_{m-1}^{ \pm 1}$ cancelled in $\varrho\left(w\left(a_{m-1}\right)\right)$.

By Lemma 6 and Theorem 7, if $n^{\prime}>n$ then the length of $u$ is greater than the length of a minimal $n$-word by at least $6\left(n^{\prime}-n\right)$, hence $n^{\prime}=n$ and $u$ is a minimal $n$-word.

Now suppose $w$ does not have the required form. Then for some $i \geq m^{\prime}$, $a_{i}$ occurs within $u$, and therefore $a_{i}^{-1}$ also occurs in $u$. In fact more than one of each must occur in $u$ (contradicting minimality), otherwise there must be an $n$-word occurring between the single occurrences of $a_{i}$ and $a_{i}^{-1}$ in $u$, giving a contradiction. 
COROLlary 12. If $n<m$ then the length of any mn-word is strictly greater than the length of a minimal Brunnian n-word.

From our knowledge of minimal Brunnian words and Lemma 11 we immediately deduce:

TheOREM 13. A minimal pq-word has length $L=2(p-q)+2^{m}\left(3 k+2^{m}\right)$ where $q=2^{m}+k$ and $k<2^{m}$.

Proof. Follows immediately from Theorem 7 and Lemma 11.

5. Braids. It seems natural to view a simple Brunnian link as a closed braid. In this section we briefly discuss this relationship which we will use in the following section to investigate when two Brunnian words represent the equivalent links that differ only in the order of the component loops.

Call a braid on $n$ threads an $n$-braid and denote the threads in an $n$-braid by $t_{0}, t_{1}, \ldots, t_{n-1}$. Let $B_{n}$ be the group of all braids on $n$ threads, so $B_{n}$ has letters $\sigma_{1}, \ldots, \sigma_{n-1}$ and defining relations

$$
\begin{aligned}
\sigma_{i} \sigma_{k} & =\sigma_{k} \sigma_{i}, \quad k \neq i-1, i+1, \\
\sigma_{i} \sigma_{i+1} \sigma_{i} & =\sigma_{i+1} \sigma_{i} \sigma_{i+1} .
\end{aligned}
$$

See $[\mathrm{H}]$ for definitions of all standard braid terms used in this section.

The closure of a braid is a link, and any link is equivalent to a closed braid [A]. Two braids define equivalent oriented links if and only if there is a finite sequence of moves involving adding or deleting a thread which shows up as a free factor $\sigma_{n-1}^{ \pm 1}$ or by conjugation, taking one braid to the other (this was first stated in $[\mathrm{M}]$ and later proved in $[\mathrm{B}]$ ).

Simple Brunnian links have an obvious braid representation, and Brunnian words correspond naturally to certain braid words. It is easy to find a closed braid equivalent to any given simple Brunnian $n$-link, $L$. Take a word in $B_{\mathrm{A}}(n-1)$ representing $L$. Think of the braid obtained by running $n-1$ straight arcs vertically, and threading $t_{0}$ through them in the obvious way. If $w=a_{i_{0}}^{\varepsilon_{0}} a_{i_{1}}^{\varepsilon_{1}} \ldots a_{i_{m}}^{\varepsilon_{m}}$, each $\varepsilon_{h}= \pm 1$, let $t_{0}$ run across the top of the threads to $t_{i_{0}}$, loop around it by passing over the top and back underneath if $\varepsilon_{0}=1$, or passing underneath and back over the top if $\varepsilon_{0}=-1$, and return back across the top. Repeat for $a_{i_{1}}$ etc. Hence if $w \in B_{\mathrm{A}}(n)$, then a braid whose closure is equivalent to the link represented by $w$ may be obtained by replacing every occurrence of $a_{i}$ in $w$ by $\sigma_{0} \sigma_{1} \ldots \sigma_{i} \sigma_{i} \sigma_{i-1}^{-1} \ldots \sigma_{0}^{-1}$, and every occurrence of $a_{i}^{-1}$ by $\sigma_{0} \ldots \sigma_{i}^{-1} \sigma_{i}^{-1} \sigma_{i-1}^{-1} \ldots \sigma_{0}^{-1}$. We call a closed braid of this form canonical. For example the 2 -word $a_{0} a_{1} a_{0}^{-1} a_{1}^{-1}$ represents a link equivalent to the canonical braid $\sigma_{0} \sigma_{0} \sigma_{0} \sigma_{1} \sigma_{1} \sigma_{0}^{-1} \sigma_{0}^{-1} \sigma_{0}^{-1} \sigma_{0} \sigma_{1}^{-1} \sigma_{1}^{-1} \sigma_{0}^{-1}$. The closure of the braid $\sigma_{0} \sigma_{0} \sigma_{0} \sigma_{1} \sigma_{1} \sigma_{0}^{-1} \sigma_{0}^{-1} \sigma_{1}^{-1} \sigma_{1}^{-1} \sigma_{0}^{-1}$ is equivalent to the link represented by $a_{0} a_{1} a_{0}^{-1} a_{1}^{-1}$. 
Let $B_{\mathrm{B}}(n)$ be the set of $n$-braids whose closures are equivalent to simple Brunnian $n$-links and call these Brunnian braids. To simplify the expression of a Brunnian braid, let $\beta_{i}=\sigma_{0} \sigma_{1} \ldots \sigma_{i} \sigma_{i} \sigma_{i-1}^{-1} \ldots \sigma_{0}^{-1}$ for each $i$. Given a canonical Brunnian braid $\beta_{i_{0}}^{\varepsilon_{0}} \beta_{i_{1}}^{\varepsilon_{1}} \ldots \beta_{i_{m}}^{\varepsilon_{m}}$, the corresponding Brunnian word is then $a_{i_{0}}^{\varepsilon_{0}} a_{i_{1}}^{\varepsilon_{1}} \ldots a_{i_{m}}^{\varepsilon_{m}} \in B_{\mathrm{A}}(n)$.

6. Equivalent Brunnian words. The objective of this section is to investigate when two Brunnian words give rise to topologically equivalent simple Brunnian links. Each Brunnian word represents a Brunnian link in canonical form. Hence $n-1$ loops are concentric about the origin and all loops have an anti-clockwise direction. It is clear, for example, that conjugate Brunnian words yield links which are topologically equivalent. The following theorem describes algebraic operations that yield topologically equivalent Brunnian links.

TheOREm 14. Suppose $v$ and $w$ are two Brunnian $n$-words and there is a sequence $w_{1}, \ldots, w_{m}$ such that $v=w_{1}, w=w_{m}$ and $w_{j+1}$ can be obtained from $w_{j}$ by an operation of one of the following types:

(i) replace $w$ by $a_{i}^{k} w a_{i}^{-k}$ for some $i<n$ and $k= \pm 1$;

(ii) replace all occurrences of $a_{i}^{ \pm 1}$ with $\left(a_{i}^{-1} a_{i+1} a_{i}\right)^{ \pm 1}$ and $a_{i+1}^{ \pm 1}$ with $a_{i}^{ \pm 1}$ (or symmetrically in $i$ and $i+1$ );

(iii) rewrite the word in the form $\delta_{0} a_{0}^{\varepsilon_{0}} \delta_{0}^{-1} \delta_{1} a_{0}^{\varepsilon_{1}} \delta_{1}^{-1} \ldots \delta_{m} a_{0}^{\varepsilon_{m}} \delta_{m}^{-1}$ such that for each $h, a_{0}^{-1}$ does not occur in $\delta_{h}$ and $\delta_{h} \neq e$, and replace each $\delta_{h} a_{0}^{\varepsilon_{h}} \delta_{h}^{-1}$ with $\delta_{h}^{-1} a_{0}^{\varepsilon_{h}} \delta_{h}$.

Then $v$ and $w$ generate equivalent links.

To prove this theorem we will exploit the connection between braids and links. Each operation corresponds to a straightforward topological operation. Since our motivation is to examine when Brunnian words represent equivalent simple Brunnian links, we are only concerned with canonical Brunnian braids. We describe the topological operation in each case, and then compute the algebraic equivalent to the topological operations.

(i) Conjugation is obvious.

(ii) This relates to swapping two adjacent curves, neither of which is the distinguished curve.

Suppose $L$ is a canonical simple Brunnian $n$-link and $B$ is the corresponding braid. Let $C_{i}$ refer to the simple closed curve which is the closure of $t_{i}$. Obtain $B^{\prime}$ from $B$ by swapping $t_{i}$ and $t_{i+1}$. We consider two possible cases. Either $t_{i}$ passes over the top of $t_{i+1}$, or it passes under it. This relates to $C_{i+1}$ passing through the inside of $C_{i}$, or $C_{i}$ passing through the inside of $C_{i+1}$ (respectively). 
Suppose first that $t_{i}$ passes under $t_{i+1}$. Define $T: B_{\mathrm{B}}(n) \rightarrow B_{\mathrm{B}}(n)$ as follows: If $B \in B_{\mathrm{B}}(n)$, take $\sigma_{i} B \sigma_{i}^{-1}$ and pull the threads $t_{i}$ and $t_{i+1}$ tight to get $B^{\prime}$. Let $T(B)$ be the canonical braid equivalent to $B^{\prime}$. Then $T$ induces a function, $T^{*}$, on the strings $\beta_{i}$ occurring in braid words. $T^{*}$ has no effect on $\beta_{j}$ if $j \neq i, i+1$. Since $T$ takes $\sigma_{i} \sigma_{i+1} \sigma_{i+1} \sigma_{i}^{-1}$ to $\sigma_{i} \sigma_{i}$, it follows that $T^{*}\left(\beta_{i+1}\right)=\left(\beta_{i}\right)$ (see Figure 2$)$.

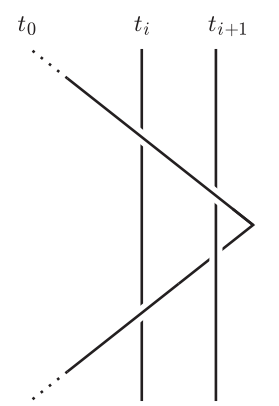

$\beta_{i+1}$

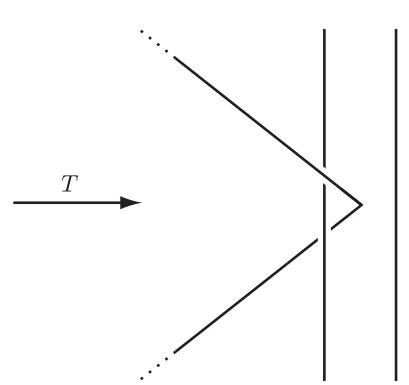

$\beta_{i}$

Fig. 2

Moreover, $T$ takes $\sigma_{i} \sigma_{i}$ to $\sigma_{i}^{-1} \sigma_{i+1} \sigma_{i+1} \sigma_{i}$ and hence $T^{*}\left(\beta_{i}\right)=\beta_{i}^{-1} \beta_{i+1} \beta_{i}$ (see Figure 3).

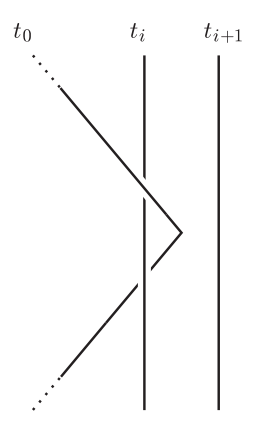

$\beta_{i}$

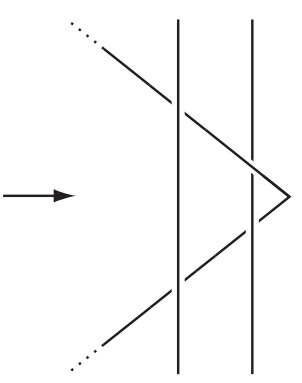

$\beta_{i+1}$

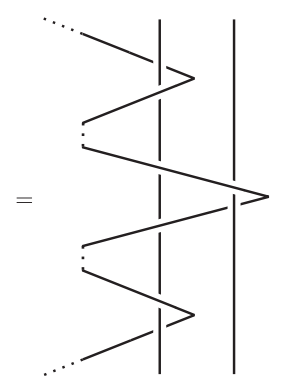

$\beta_{i}^{-1} \beta_{i+1} \beta_{i}$

Fig. 3

For example if $t_{1}$ passes under $t_{2}$ and $B=\beta_{0} \beta_{1}^{-1} \beta_{0}^{-1} \beta$, then $T(B)=$ $\beta_{0}^{-1} \beta_{1} \beta_{0} \beta_{1}^{-1}$. Thus the words $a_{0} a_{1} a_{0}^{-1} a_{1}^{-1}$ and $a_{0}^{-1} a_{1} a_{0} a_{1}^{-1}$ define equivalent links.

Suppose now that $t_{i}$ passes over $t_{i+1}$. Define $T: B_{\mathrm{B}}(n) \rightarrow B_{\mathrm{B}}(n)$ similarly to the above, but taking $\sigma_{i}^{-1} B \sigma_{i}$ rather than $\sigma B \sigma^{-1}$. Again $T^{*}$ has no effect on $\beta_{j}$ if $j \neq i, i+1$, while $T^{*}\left(\beta_{i}\right)=\beta_{i+1}$ and $T^{*}\left(\beta_{i+1}\right)=\beta_{i+1} \beta_{i} \beta_{i+1}^{-1}$. 
For example if $t_{1}$ passes over $t_{2}$ and $B=\beta_{0} \beta_{1} \beta_{0}^{-1} \beta_{1}^{-1}$, then $T(B)=$ $\beta_{1} \beta_{1} \beta_{0} \beta_{1}^{-1} \beta_{0}^{-1} \beta_{1}^{-1}$.

(iii) This relates to swapping the distinguished curve with $C_{0}$.

Suppose the closure of $B \in B_{\mathrm{B}}(n)$ is $L \in B_{\mathrm{S}}(n)$. Since we assume that $B$ is canonical, $t_{0}$ corresponds to the distinguished curve in $L$. Let $B^{\prime \prime}$ be a braid with closure equivalent to $L$, but with $t_{1}$ as the distinguished curve. We will take $B^{\prime \prime}$ to be the braid derived from $B$ by pulling the threads $t_{0}, t_{2}, t_{3}, \ldots, t_{n-1}$ taut, and letting $t_{1}$ loop around them. Then $\sigma_{0} B^{\prime \prime} \sigma_{0}^{-1}$ swaps $t_{0}$ and $t_{1}$. Let $B^{\prime}$ be the canonical braid equivalent to $\sigma_{0} B^{\prime \prime} \sigma_{0}^{-1}$. The process we will now describe will combine these two steps and transform $B$ directly into $B^{\prime}$.

Suppose $B=\beta_{i_{0}}^{\varepsilon_{0}} \beta_{i_{1}}^{\varepsilon_{1}} \ldots \beta_{i_{l}}^{\varepsilon_{l}}$ and consider the corresponding word $w=$ $a_{i_{0}}^{\varepsilon_{0}} a_{i_{1}}^{\varepsilon_{1}} \ldots a_{i_{l}}^{\varepsilon_{l}} \in B_{\mathrm{A}}(n)$. We will construct a word $w^{\prime}$ which reduces to $w$, with the form $\delta_{0} a_{0}^{\varepsilon_{0}} \delta_{0}^{-1} \delta_{1} a_{0}^{\varepsilon_{1}} \delta_{1}^{-1} \ldots \delta_{m} a_{0}^{\varepsilon_{m}} \delta_{m}^{-1}$ such that for each $h, a_{0}^{-1}$ does not occur in $\delta_{h}$ and $\delta_{h} \neq e$.

At least one occurrence of $a_{0}^{\varepsilon}$ in $w$ is flanked by some $a_{i}$ and $a_{i}^{-1}$. For the $p$ th occurrence of $a_{0}^{\varepsilon}$ (possibly a string of length greater than 1) in $w$, let $\alpha_{p}$ be the maximal string such that $a_{0}^{ \pm 1}$ does not occur in $\alpha_{p}$ and $a_{0}^{\varepsilon}$ occurs in the string $\gamma_{0 p}=\alpha_{p} a_{0}^{ \pm 1} \alpha_{p}^{-1}$. If $w=\gamma_{00} \gamma_{01} \cdots \gamma_{0 m}$ we are done, otherwise for each possible $p$ let $\alpha_{1 p}$ be the maximal string such that $a_{0}^{ \pm 1} \notin \alpha_{1 p}$ and $\alpha_{1 p} \gamma_{0 q} \gamma_{0(q+1)} \cdots \gamma_{0 l_{p}} \alpha_{1 p}^{-1}$ occurs in $w$ (at least one such string occurs for cancelling to proceed in $\left.\varrho\left(w\left(-a_{0}\right)\right)\right)$. Let $w_{1}$ be the word obtained from $w$ by replacing each maximal string $\alpha_{1 p} \gamma_{0 q} \gamma_{0(q+1)} \cdots \gamma_{0 l} \alpha_{1 p}^{-1}$ by

$$
\gamma_{1 p}=\alpha_{1 p} \gamma_{0 q} \alpha_{1 p}^{-1} \alpha_{1 p} \gamma_{0(q+1)} \alpha_{1 p}^{-1} \ldots \alpha_{1 p} \gamma_{0 l} \alpha_{1 p}^{-1} .
$$

Note that $w_{1}$ reduces to $w$.

Now change strings $\alpha_{2 p} \gamma_{1 q} \gamma_{1(q+1)} \cdots \gamma_{1 l} \alpha_{2 p}^{-1}$ in $w_{1}$, where $\alpha_{2 p}$ are maximal, to

$$
\gamma_{2 p}=\alpha_{2 p} \gamma_{1 q} \alpha_{2 p}^{-1} \alpha_{2 p} \gamma_{1(q+1)} \alpha_{2 p}^{-1} \ldots \alpha_{2 p} \gamma_{1 l} \alpha_{2 p}^{-1}
$$

if $w_{1} \neq \gamma_{10} \gamma_{11} \ldots \gamma_{1 m}$. Continue until $w_{m}=\gamma_{m 0} \gamma_{m 1} \ldots \gamma_{m l}$. Note that $w_{m}$ reduces to $w$ and has the form $\delta_{0} a_{0}^{\varepsilon_{0}} \delta_{0}^{-1} \delta_{1} a_{0}^{\varepsilon_{1}} \delta_{1}^{-1} \ldots \delta_{m} a_{0}^{\varepsilon_{m}} \delta_{m}^{-1}$ as required. Then $w^{\prime}=w_{m}$.

Now consider the braid corresponding to $w^{\prime}$. If $\alpha$ is a string in a Brunnian word $w$ denote the corresponding string in the canonical braid word representing $w$ by $b(\alpha)$. We can unravel each $b\left(\delta_{p}\right) \beta_{0} b\left(\delta_{p}^{-1}\right)$ to get $b\left(\delta_{p}^{-1}\right) \beta_{0} b\left(\delta_{p}\right)$. Each $b\left(\delta_{p}\right) \beta_{0} b\left(\delta_{p}^{-1}\right)$ is a symmetrical bit of the woven thread $t_{0}$, which picks up $t_{1}$ at the very centre. By unravelling it, $t_{1}$ is pulled through following $b\left(\delta_{p}\right)$ (and $b\left(\delta_{p}^{-1}\right)$ ), but in the opposite direction. Turning $t_{1}$ into the first thread corresponds to conjugation by $\sigma_{0}$. Thus the final outcome is a replacement of each $b\left(\delta_{p}\right) \beta_{0} b\left(\delta_{p}^{-1}\right)$ with $b\left(\delta_{p}^{-1}\right) \beta_{0} b\left(\delta_{p}\right)$ and conjugation by $\sigma_{0}$. 
Pulling threads other than $t_{1}$ taut and conjugating by $\sigma_{0}$ does not give a canonical braid. It would be necessary to strategically add strings $\sigma_{i}^{-1} \sigma_{i-1} \ldots \sigma_{0}^{-1} \sigma_{0} \sigma_{1} \ldots \sigma_{i}$ to the braid. However, this process is incorporated in the algebra.

For example, consider

$$
B=\beta_{0} \beta_{1} \beta_{0}^{-1} \beta_{1}^{-1} \beta_{2} \beta_{1} \beta_{0} \beta_{1}^{-1} \beta_{0}^{-1} \beta_{2}^{-1} .
$$

Then

$$
\begin{aligned}
w & =a_{0} a_{1} a_{0}^{-1} a_{1}^{-1} a_{2} a_{1} a_{0} a_{1}^{-1} a_{0}^{-1} a_{2}^{-1}, \\
w_{1} & =(e) a_{0}(e) \cdot\left(a_{1}\right) a_{0}^{-1}\left(a_{1}^{-1}\right) \cdot a_{2} \cdot\left(a_{1}\right) a_{0}\left(a_{1}^{-1}\right) \cdot(e) a_{0}^{-1}(e) \cdot a_{2}^{-1}, \\
w_{2} & =(e) a_{0}(e) \cdot\left(a_{1}\right) a_{0}^{-1}\left(a_{1}^{-1}\right) \cdot\left(a_{2}\right)\left(a_{1}\right) a_{0}\left(a_{1}^{-1}\right)\left(a_{2}^{-1} a_{2}\right)(e) a_{0}^{-1}(e)\left(a_{2}^{-1}\right) \\
& =(e) a_{0}(e) \cdot\left(a_{1}\right) a_{0}^{-1}\left(a_{1}^{-1}\right) \cdot\left(a_{2} a_{1}\right) a_{0}\left(a_{1}^{-1} a_{2}^{-1}\right) \cdot\left(a_{2} e\right) a_{0}^{-1}\left(e a_{2}^{-1}\right) \\
& =w^{\prime},
\end{aligned}
$$

and

$$
\begin{aligned}
B^{\prime} & =(e) \beta_{0}(e) \cdot\left(\beta_{1}^{-1}\right) \beta_{0}^{-1}\left(\beta_{1}\right) \cdot\left(\beta_{1}^{-1} \beta_{2}^{-1}\right) \beta_{0}\left(\beta_{2} \beta_{1}\right)\left(\beta_{2}^{-1} e\right) \beta_{0}^{-1}\left(e \beta_{2}\right) \\
& =\beta_{0} \beta_{1}^{-1} \beta_{0}^{-1} \beta_{1} \beta_{1}^{-1} \beta_{2}^{-1} \beta_{0} \beta_{2} \beta_{1} \beta_{2}^{-1} \beta_{0}^{-1} \beta_{2} .
\end{aligned}
$$

Figure $4(\mathrm{a})$ is the braid $\beta_{0} \beta_{1} \beta_{0}^{-1} \beta_{1}^{-1}$. Changing the distinguished loop gives $4(\mathrm{~d})$, the braid $\beta_{0} \beta_{1}^{-1} \beta_{0}^{-1} \beta_{1}$. Figures $4(\mathrm{~b})$ and $4(\mathrm{c})$ indicate how $t_{1}$ is pulled through as $t_{0}$ unravels.

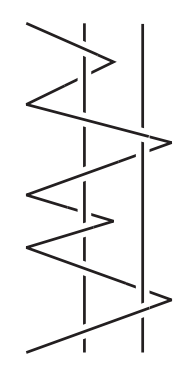

(a)

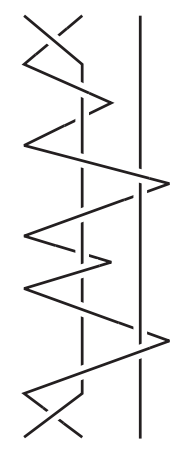

(b)

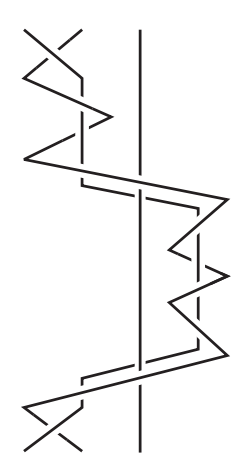

(c)

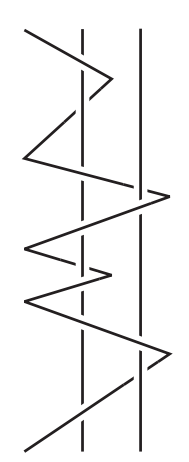

(d)

Fig. 2. Changing the distinguished loop

\section{References}

[A] J. W. Alexander, A lemma on systems of knotted curves, Proc. Nat. Acad. Sci. U.S.A. 9 (1923), 93-95.

[B] J. S. Birman, Braids, Links, and Mapping Class Groups, Ann. of Math. Stud. 82, Princeton Univ. Press, 1975. 
[H] V. L. Hansen, Braids and Coverings, Cambridge Univ. Press, 1989.

[M] A. Markoff, $\ddot{U}$ ber die freie Äquivalenz der geschlossenen Zöpfe, Mat. Sb. 1 (1936), 73-78.

Department of Mathematics

University of Auckland

University of Pittsburgh

Private Bag 92019

Pittsburgh, PA 15260, U.S.A.

Auckland, New Zealand

E-mail: gartside@math.pitt.edu

E-mail: s.greenwood@auckland.ac.nz

Received 6 June 2006;

in revised form 1 December 2006 